\title{
THE STATE OF DENTISTS' KNOWLEDGE ABOUT THE RELATIONSHIP BETWEEN TEMPOROMANDIBULAR DISORDERS AND LYME DISEASE SYMPTOMS
}

\author{
Magdalena Osiewicz' ', Grażyna Biesiada², Jolanta Pytko-Polończyk \\ 'Department of Integrated Dentistry, Jagiellonian University, Medical College, Krakow, Poland \\ ${ }^{2}$ Department of Infectious and Tropical Diseases, Faculty of Medicine, Jagiellonian University Medical College, Krakow, Poland
}

\begin{abstract}
INTRODUCTION: Dentists may be the first to whom the patient with Lyme disease (LD) reports symptoms of temporomandibular disorders (TMD). For this reason, it is important that dentists should be aware of the relationship between LD and TMD.

ОвJectives: Aim of this study was to assess dentists' knowledge about the relationship between LD and TMD. MATERIAL AND METHODS: A group of 201 dentists participating in the project were volunteers, who came from randomly selected dental offices. All the participants anonymously completed a questionnaire, in which they were asked three questions concerning TMD and LD.

ReSults: Among dentists, $81.1 \%$ agreed that LD symptoms could mimic TMD, and $81.1 \%$ of the respondents thought that fluctuating pain in the area of temporomandibular joint and/or myofascial muscles imitate TMD. Among participants, $37.8 \%$ indicated limited mouth opening, whereas $19.4 \%$ of respondents marked sounds as symptoms. There was a significant relationship $(p<0.05)$ between those dentists who knew that LD symptoms can mimic TMD, as they considered the possibility of Lyme disease more often than others.

ConcLusions: The results of this study indicate that dentists' knowledge of tick and tick-borne diseases is sufficient, and it would be a relevant public health strategy as it could reduce the risk of unnecessary TMD treatment for patients suffering from $\mathrm{LD}$.
\end{abstract}

KEY WORDS: Lyme disease, questionnaire, temporomandibular disorders, TMD, dentist.

J Stoma 2020; 73, 1: 32-35

DOI: https://doi.org/10.5114/jos.2020.94175

\section{INTRODUCTION}

In Central and Eastern Europe, Lyme disease (LD) is a tick-borne infection caused by bacteria of Borrelia species, classified as $B$. burgdorferi $(\mathrm{Bb})$ strain, which is transmitted by ticks, Ixodesricinus and Ixoduspersculatus $[1,2]$. The course of the disease can be divided into two periods: an early one, which can be localized or disseminated, and a late (chronic) period $[2,3]$.
The main LD clinical symptom in the early localized period is the erythema migrans (EM), which is a pathogenic symptom experienced by $80 \%$ of $\mathrm{Bb}$-infected patients [2-4]. In the period of early dissemination, symptoms of neuroborreliosis and inflammatory arthritis are likely to occur. Neuroborreliosis manifests itself as a cranial nerve palsy, nerve root palsy, and meningitis $[1,2,4]$. Inflammatory arthritis most often affects large joints, especially knee joints. LD symptoms occurring

JOURNAL OF STOMATOLOGY CZASOPISMO STOMATOLOGICZNE

AdDress for Correspondence: Magdalena Osiewicz, PhD, Department of Integrated Dentistry, Jagiellonian University, Medical College, Krakow, Montelupich, 31-155 Kraków, Poland, e-mail: magdalena.osiewicz@uj.edu.pl

ReCEIVED: 12.01.2020 • ACCEPTED: 05.03.2020 • PUBLISHED: 25.03.2020 
later are chronic inflammatory arthritis, chronic neuroborreliosis, or chronic atrophic dermatitis $[1,2,4,5]$. Apart from EM, LD symptoms are not characteristic for the infection, and blood tests are needed to confirm the diagnosis. Unfortunately, in the early periods, $\mathrm{Bb}$ antibodies are not present in blood yet. Therefore, early LB symptoms such as pain might be misleading for a dentist, who is often the general practitioner of patient, whose symptoms may resemble temporomandibular disorders (TMD). In Poland, in 2018, there were 20,139 new cases of LD, and the prevalence rate was estimated at 52.4 cases per 100,000 inhabitants. In the population residing in endemic regions in Europe, an increased level of Borrelia antibodies has been found in 3 to $15 \%$ of population, whereas in risk groups (such as foresters or ornithologists), the number varied between 20 and $40 \%$ [6].

TMD are characterized by the presence of several symptoms such as pain in the masticatory muscles and/ or temporomandibular joint (TMJ), limited jaw movements, and TMJ noises (i.e. clicking and/or crepitus) during function [7]. The Research Diagnostic Criteria for Temporomandibular Disorders (RDC/TMD) and their updated version (i.e. the Diagnostic Criteria for Temporomandibular Disorders DC/TMD) is a diagnostic standard in both clinical tests and research studies [8-10]. Orofacial pain occurs in $10 \%$ of human population [11-13]. It is important that all dentists should be aware of the relationship between LD and TMD, as it is often a dentist whom the patient initially consults. It would be helpful if the dentist could refer the patient to the right specialist for a diagnosis and LD treatment, which in turn would limit delays in medical intervention and/or unnecessary treatment. Therefore, the aim of this study was to assess dentists' knowledge of the relationship between LD and TMD.

TABLE 1. Qualitative variable percentages of questions 1,2 , and 3

\begin{tabular}{|c|c|c|}
\hline \multicolumn{2}{|c|}{ Question } & $n(\%)$ \\
\hline \multicolumn{3}{|c|}{1} \\
\hline & Symptoms of Lyme disease can mimic TMD & $163(81.1)$ \\
\hline & Symptoms of Lyme disease do not mimic TMD & $30(14.9)$ \\
\hline & No answer & $8(3.9)$ \\
\hline \multicolumn{3}{|c|}{$2^{*}$} \\
\hline & Fluctuating pain of TMD & $163(81.1)$ \\
\hline & Mouth opening limitation & $76(37.8)$ \\
\hline & Joint sounds & $39(19.4)$ \\
\hline \multicolumn{3}{|c|}{3} \\
\hline & I consider Lyme disease & $70(34.8)$ \\
\hline & I do not consider Lyme disease & $119(59.2)$ \\
\hline & No answer & $12(6.0)$ \\
\hline
\end{tabular}

*Multiple answers

TMD - temporomandibular disorders

\section{MATERIAL AND METHODS}

A group of dentists participating in the project were volunteers, who came from randomly selected dental surgeries. All participants had completed their medical studies in Poland. Prior to the research, all participating dentists had been informed about the aim of the study and signed a formal agreement to participate. The research had been approved by the Bioethical Commission of the Jagiellonian University (No KABET 1072.6120.83.2018) and was carried out in accordance with the Helsinki Declaration between April 2018 and August 2019. All participants anonymously completed a questionnaire, in which they were asked three questions concerning TMD and LD. Dentists completed the questionnaire next to the examiners. The first question was: "To the best of your knowledge, do you think Lyme disease symptoms may resemble temporomandibular disorders?". The participants were to choose either "yes" or "no" answer. The second question, which was a multiple-choice question, was answered only by the responders who answered "yes" to the first question: "Which of the Lyme disease symptoms may resemble temporomandibular disorders?". Here, the participants chose one of the following: 1. Fluctuating pain in the temporomandibular joint area and/or myofascial muscles; 2. Limited mouth opening; 3. Sounds in the joint. The third question concerned only the cases, where there was a suspicion of LD. It required a "yes" or "no" answer: "If the TMD still continue in spite of the treatment you have prescribed, do you - on the basis of the patient's medical history - take into consideration a possibility that the patient might be suffering from Lyme disease?”.

\section{STATISTICAL ANALYSIS}

Qualitative variable percentages and raw counts were reported. Comparisons of qualitative variables in groups were conducted with $\chi^{2}$ test (with Yates' correction for $2 \times 2$ tables) or with Fisher's exact test, where expected values were low. Analyses were conducted at 0.05 level of significance. R software, version 3.6.1 was used [14].

\section{RESULTS}

Total of 201 volunteers answered the questionnaire, and all of them were included in the project. Among dentists, $81.1 \%$ agreed that LD symptoms could resemble those of TMD. Moreover, $81.1 \%$ of the respondents thought that fluctuating pain in the area of temporomandibular joint and/or myofascial could resemble TMD pain. $37.8 \%$ of participants chose limited mouth opening, whereas $19.4 \%$ of respondents selected sounds as possible symptoms (Table 1 ). There was a significant relationship $(p<0.05)$ between those dentists who knew that 
TABLE 2. Statistical analysis between question 1 and 3

\begin{tabular}{l|c|c|c|}
\hline \multicolumn{2}{|l|}{$\begin{array}{c}\text { Symptoms of Lyme disease can mimic TMD } \\
(\boldsymbol{n}=163)\end{array}$} & $\begin{array}{c}\text { Symptoms of Lyme disease does not mimic TMD } \\
(\boldsymbol{n}=30)\end{array}$ \\
\hline I consider Lyme disease & $66(40.49 \%)$ & $3(10.00 \%)$ \\
\hline I do not consider Lyme disease & $89(54.60 \%)$ & $25(83.33 \%)$ \\
\hline No answer & $8(4.91 \%)$ & $2(6.67 \%)$ \\
\hline$p-$ Fisher's exact test
\end{tabular}

$T M D$ - temporomandibular disorders

LD symptoms could mimic TMD, as they considered the possibility of LD more often than others (Table 2).

\section{DISCUSSION}

The aim of our study was to assess dentists' level of awareness of interdependence between TMD and LD. This is probably the first research of this kind in the world. The results of this project showed that dentists who were aware that some LD symptoms could mimic those of TMD, did consider the possibility of Lyme disease and were responsible for their patients' problems more often than those who were not aware of the similarities. Moreover, $81.1 \%$ of the respondents were aware of the interdependence mentioned above; therefore, the level of their knowledge was assessed as good. The respondents' knowledge of LD symptoms, which can imitate various TMD symptoms was also high.

The spread of tick-borne infectious diseases constitutes a rapidly growing health risk in Europe and elsewhere [15]. In case of some patients, LD symptoms are mild or absent. However, for some individuals they can be severe, especially if they are not recognized and treated early enough [1]. Regardless of the form LD may take, except for EM, the patient's blood needs to be tested in a two-step procedure to confirm the presence of antibodies against Borrelia burgdorferi. The first is Elisa test class IgG and IgM, and if the result is positive or borderline, it needs to be confirmed by a Western-blot test $[1,3,5]$. Unfortunately, at the early LD stages, no Bb may be detectable in the patient's blood. Moreover, LD symptoms may resemble several TMD symptoms, so the dentists' knowledge and their perception of the risk related to tick bites and tick-borne diseases is very important $[16,17]$. As far as TMD occurrence in LD patients is concerned, the research by Osiewicz et al. shows that $70 \%$ of LD patients tested positive for one of the RDC/TMD symptoms, namely myofascial pain [16]. It is possibly due to the fact that the presence of comorbid condition causes widespread muscle sensitization. However, according to the earlier studies by Koutris et al. and Vischer et al., differential diagnosis between TMD and comorbid diseases should be made by using function-dependent tests $[18,19]$. The aim of the function-dependent tests is to elicit muscle and/or temporomandibular joint pain, either during mandibular movement (i.e. dynamic) and/ or static muscle contractions. If the tests are negative, the patient should be immediately referred to an infectious disease specialist to avoid wasting valuable time on any unnecessary treatment, and to prevent further delay in choosing an appropriate intervention.

If the questionnaires are well-designed and conducted appropriately, they can be a source of wide knowledge, clarifying many issues, and providing answers to the research questions. However, a questionnaire presents a risk of including questions, which may suggest the answers the researcher expects, even when the responder is trying to be absolutely neutral. The limitation of this study is that certain questions might have suggested particular practices or actions, which some of the respondents had not undertaken at all.

\section{CONCLUSIONS}

The results of this study indicate that dentists' knowledge of tick and tick-borne diseases is sufficient, and it would be relevant for public health strategy, as it could reduce the risk of unnecessary TMD treatment for patients suffering from LD.

\section{ACKNOWLEDGEMENTS}

The authors would like to thank Dr Anna Maciąg and Students (Paulina Kojat, Maria Gut, and Zuzanna Kazibudzka) from the Scientific Group of Integrated Dentistry at the Department of Integrated Dentistry, Dental Institute, Jagiellonian University, Medical College, Krakow, for their help in collecting the questionnaires.

\section{CONFLICT OF INTEREST}

The authors declare no potential conflicts of interest with respect to the research, authorship, and/or publication of this article.

\section{References}

1. Biesiada G, Czepiel J, Leśniak MR, Garlicki A, Mach T. Lyme disease: review. Arch Med Sci 2012; 8: 978-982.

2. Lantos P, Rumbaugh J, Bockenstedt L, et al. Draft clinical practice guidelines by the Infectious Diseases Society of America (IDSA), 
American Academy of Neurology (AAN), and American College of Rheumatology (ACR): Guidelines for the Prevention, Diagn. 2019. Available at: https://view.protectedpdf.com/ad6GFZ (Accessed: 5.02.2020).

3. Pancewicz S, Moniuszko-Malinowska A, Garlicki A, Grygorczuk S, Czupryna P. Diagnostyka i leczenie boreliozy z Lyme. Standardy Polskiego Towarzystwa Epidemiologicznego i Lekarzy Chorób Zakaźnych. Rekom. PTEILCHZ. 2018. Available at: http://www. pteilchz.org.pl/informacje/rekomendacje/ (Accessed: 5.02.2020).

4. Stanek G, Wormser GP, Gray J, Strle F. Lyme borreliosis. Lancet 2012; 379: 461-473.

5. Borchers AT, Keen CL, Huntley AC, Gershwin ME. Lyme disease: a rigorous review of diagnostic criteria and treatment. J Autoimmun 2015; 57: 82-115.

6. Gryczynska A, Welc-Faleciak R. Long-term study of the prevalence of Borrelia burgdorferi s.l. infection in ticks (Ixodes ricinus) feeding on blackbirds (Turdus merula) in NE Poland. Exp Appl Acarol 2016; 70: 381-934.

7. deLeeuw R KG. Differential diagnosis and management of TMDs. $6^{\text {th }}$ ed. Hanover Park: Quintessence Publishing Co, Inc; 2018.

8. Dworkin SF, LeResche L. Research diagnostic criteria for temporomandibular disorders: review, criteria, examinations and specifications, critique. J Craniomandib Disord 1992; 6: 301-355.

9. Schiffman E, Ohrbach R, Truelove E, et al. Diagnostic criteria for temporomandibular disorders (DC/TMD) for clinical and research applications: recommendations of the International RDC/ TMD Consortium Network and Orofacial Pain Special Interest Group. J Oral Facial Pain Headache 2014; 28: 6-27.

10. Osiewicz M, Lobbezoo F, Loster B, Wilkosz M, Naeije M, Ohrbach R. Research diagnostic criteria for temporomandibular disorders (RDC/TMD) - The Polish version of a dual-axis system for the diagnosis of TMD. RDC/TMD Form. J Stomatol 2013; 66: 576-649.

11. Dworkin SF, Massoth DL. Temporomandibular disorders and chronic pain: sisease or illness? J Prosthet Dent 1994; 72: 29-38.

12. Loster JE, Osiewicz MA, Groch M, Ryniewicz W, Wieczorek A The prevalence of TMD in Polish young adults. J Prosthodont 2017; 26: 284-288.

13. Osiewicz MA, Lobbezoo F, Loster BW, Loster JE, Manfredini D. Frequency of temporomandibular disorders diagnoses based on RDC/ TMD in a Polish patient population. Cranio 2018; 36: 304-310.

14. R Core Team. R: A language and environment for statistical computing. R Foundation for Statistical Computing. 2019. Available at: https://www.r-project.org/ (Accessed: 5.02.2020).

15. Lindquist L, Vapalahti O. Tick-borne encephalitis. Lancet 2008; 371: 1861-1871

16. Osiewicz M, Manfredini D, Biesiada G, et al. Prevalence of function-dependent temporomandibular joint and masticatory muscle pain, and predictors of temporomandibular disorders among patients with Lyme disease. J Clin Med 2019; 8: 929.

17. Osiewicz M, Manfredini D, Biesiada G, et al. Differences between palpation and static/dynamic tests to diagnose painful temporomandibular disorders in patients with Lyme disease. Clin Oral Investig 2019; 23: 4411-4416.

18. Koutris M, Visscher CM, Lobbezoo F, Naeije M. Comorbidity negatively influences the outcomes of diagnostic tests for musculoskeletal pain in the orofacial region. Pain 2013; 154: 927-932.

19. Visscher CM, Naeije M, De Laat A, et al. Diagnostic accuracy of temporomandibular disorder pain tests: a multicenter study. J Orofac Pain 2009; 23: 108-114. 